\section{m-Tyrosin, eine neue Aminosäure aus dem Milchsaft von Euphorbia Myrsinites L. ${ }^{1}$}

Von K. Mothes, H. R. Schütte, P. Müller, M. v. Ardenne und R. Tümmler

Deutsche Akademie der Wissenschaften zu Berlin Institut für Biochemie der Pflanzen, Halle/Saale und Forschungsinstitut Manfred v. Ardenne, DresdenWeißer Hirsch

(Z. Naturforschg. 19 b, 1161-1162 [1964] ; eingeg. am 8. Oktober 1964)

In den letzten Jahren hat sich herausgestellt, daß Milchsäfte von Pflanzen verschiedenster systematischer Stellung häufig ein ungewöhnliches Aminosäurespektrum aufweisen ${ }^{2-7}$. Neben den bekannten Aminosäuren finden sich oft latexspezifische ungewöhnlicher Struktur. In vielen Fällen stellen sie die Hauptaminoverbindung des jeweiligen Milchsaftes dar, während die üblichen Aminosäuren mengenmäßig stark zurücktreten. Diese Verbindungen gehören keinem bestimmten Grundtyp an, sondern der Charakter wechselt meist von Art zu Art. In unserem Arbeitskreis wurde eine Reihe von Milchsäften papierchromatographisch auf ihre Aminosäure-Zusammensetzung geprüft ${ }^{8,9}$. Dabei sind u. a. auf den Chromatogrammen ninhydringelbe Flecke gefunden worden, die nicht bekannten Strukturen zuzuordnen waren. Das Auftreten der diesen Flecken zu Grunde liegenden Verbindungen scheint auf den Milchsaft von Euphorbia-Arten beschränkt zu sein, jedoch kommen sie auch bei diesen nicht überall vor.

Wir waren an der Klärung der Struktur dieser Verbindungen interessiert und verwendeten als Ausgangsmaterial für die Isolierung die mediterrane Euphorbia myrsinits L., die bei uns auch bei Anbau im Freiland reichlich Milchsaft liefert, in dem diese Verbindungen in einer für die Isolierung aussichtsreichen Konzentration vorkommen.

170 Pflanzen wurden kurz unter der Sproßspitze abgeschnitten und der freigesetzte Milchsaft (etwa 0,5 $l$ ) in 96-proz. Äthanol aufgefangen. Nach Abtrennen der koagulierten Polyterpene und Proteine wurde der alkoholische Extrakt über eine mit dem Kationenaustauscher Wofatit KPS gefüllte Säule geschickt. Die Elution der Aminosäuren vom Austauscher erfolgte mit 3-n. $\mathrm{NH}_{3}$ in 70-proz. Äthanol. Der nach Vertreiben des Lösungsmittel im Vakuum verbleibende Rückstand wurde in wenig $\mathrm{n}$-Propanol/Wasser $=3: 1$ aufgenommen. Nach weiterer Reinigung und Fraktionierung an Zellulosepulver-Säulen (Schleicher \& Schüll Nr. 123 a) er-

1 9. Mitt. der Reihe „Zur Biochemie des Milchsaftes"; 8. Mitt. K. Mothes u. L. Meissner, Forschungen und Fortschritte 1964 (im Druck).

2 I. Liss, Flora [Jena] 151, 351 [1961].

3 I. Liss, Naturwissenschaften 48, 304 [1961].

4 I. Liss, Phytochemistry 1, 87 [1962].

5 W. Schenk u. H. R. Sсhütte, Naturwissenschaften 48, 223 [1961].

6 Ch. Montant, Compt. Rend. Soc. biol. 150, 440 [1956].

7 Ch. Montant u. I. M. Touze-Soulet, Experientia [Basel] 19, 624 [1963]. hielten wir schließlich $120 \mathrm{mg}$ einer einheitlichen, farblosen Verbindung (Schmp. $272-274{ }^{\circ} \mathrm{C}$, Zersetzung).

Papierchromatographisch läßt sich die Verbindung nur mit Ninhydrin und auf Phenole ansprechenden Reagenzien mit Ausnahme von $\mathrm{FeCl}_{3}$ nachweisen, was auf eine phenolische Aminosäure hindeutete. Auffällig ist besonders die intensive Orangefärbung nach Kupplung mit diazotierter Sulfanilsäure und nach dem Alkalisieren. Die Lage auf dem Chromatogramm zeigt große Ähnlichkeit mit Tyrosin. Das UV-Absorptionsspektrum (Hilger-Uvispec) in Wasser stimmt ebenfalls weitgehend mit dem von Tyrosin überein: $\lambda_{\max }=$ $273 \mathrm{~m} \mu$. In $0,1-n$. $\mathrm{NaOH}$ tritt eine bathochrome Verschiebung des Maximums um $18 \mathrm{~m} \mu$ ein, während sich dieses bei Messung in 0,1-n. $\mathrm{HCl}$ nicht verändert. Die Verbindung ist in Wasser schwer, in Äthanol mäßig und in Alkali leicht löslich. Ionophoretisch erwies sie sich als neutrale Aminosäure. Das IR-Spektrum (in $\mathrm{KBr}$ ) weist $\mathrm{OH}$ - und $\mathrm{NH}_{2}-\mathrm{Banden}$ aus, zeigt das Vorliegen eines aromatischen Ringes und deutet darauf hin, daß die Verbindung stark assoziiert vorliegt * Bei Behandlung mit Säure oder Alkali erweist sich die Substanz als relativ stabil. Nach Umsetzung mit salpetriger Säure ${ }^{10}$ läßt sich die Verbindung papierchromatographisch nicht mehr nachweisen, so daß die Struktur eines primären Amins angenommen werden muß.

Die Elementaranalyse ergab folgende Werte:

$$
\begin{array}{lllllll}
\mathrm{C}_{9} \mathrm{H}_{11} \mathrm{O}_{3} \mathrm{~N} & \text { Gef. } & \text { C } 59,7 / 59,8 & \mathrm{H} & 6,4 / 6,7 & \mathrm{~N} & 7.31 \\
& \text { Ber. } & \text { C } 59,66 & \mathrm{H} & 6,07 & \mathrm{~N} & 7,73 .
\end{array}
$$

Das Massenspektrum wurde nach einer neu entwickelten Methode aufgenommen ${ }^{11}$, wobei an die Moleküle der Substanz in der Ionenquelle des Massenspektrographen negative Ladungsträger (Elektronen, Ionen) geringer kinetischer Energien angelagert werden. Das auf eine Photoplatte aufgenommene Spektrum, von dem eine mikrophotometrische Registrierung (Abb. 1) wiedergegeben wird, weist eine intensive Massenlinie bei 180 auf, die mit dem für $\mathrm{C}_{9} \mathrm{H}_{11} \mathrm{O}_{3} \mathrm{~N}$ berechneten Mol.Gew. von 181,2 gut übereinstimmt. Weitere schwächere Massenlinien liegen u. a. bei 155, 134, 119, 106 und 93 und lassen sich Fragmenten, die durch thermische Zersetzung des $m$-Tyrosins entstanden sein können, zuordnen.

Alkalischmelze im Mikromaßstab führte zu einer Verbindung, die papierchromatographisch in mehreren Laufmitteln und im Nachweis mit verschiedenen Phenolreagenzien mit $m$-Hydroxybenzoesäure übereinstimmt. Zur Klärung der Struktur der Seitenkette

8 W. Schenk, Diplomarbeit, Universität Halle, Math.-Nat. Fak. 1959.

9 I. Liss, Dissertation, Universität Halle, Math.-Nat. Fak. 1962.

* Herrn Dr. Kolbe, Chemische Institute der Universität Halle, danken wir für Aufnahme und Diskussion des IR. Spektrums.

10 B. Wiткор u. C. M. Foltz, J. Amer. chem. Soc. 79, 192 [1957].

11 M. v. Ardenne, K. Steinfelder u. R. Tümmler, Experientia [Basel] 19, 178 [1963]. 

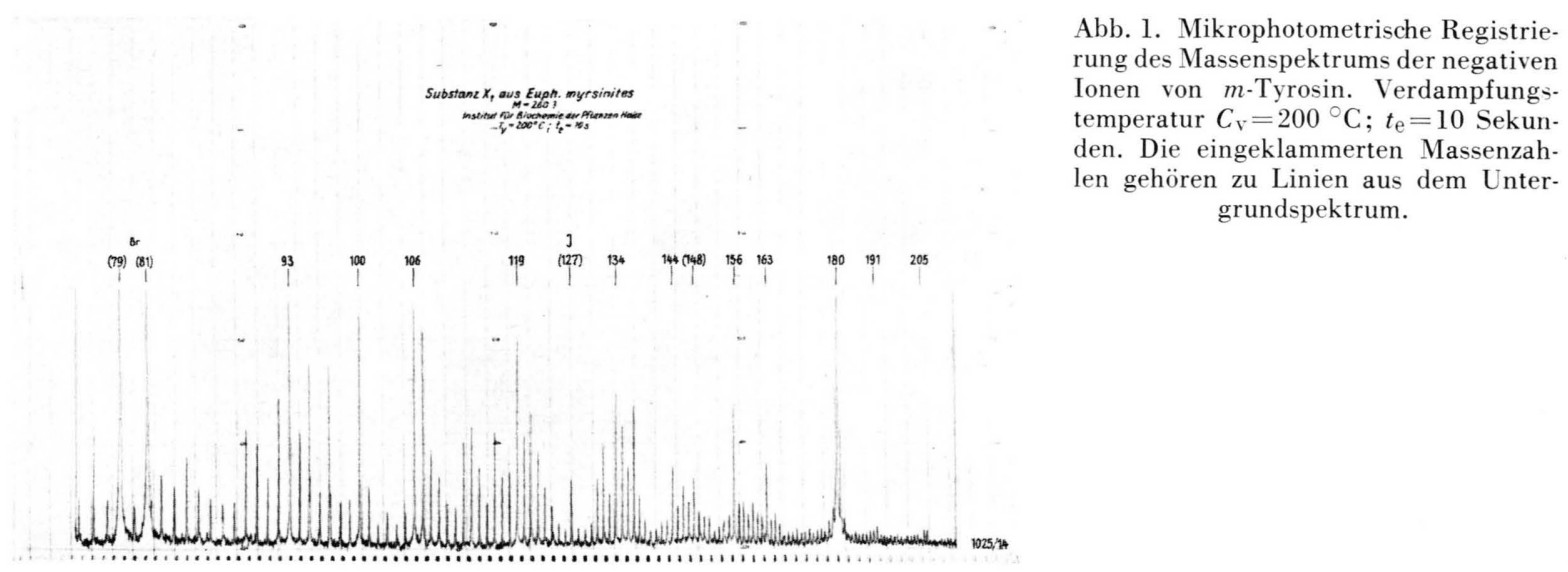
grundspektrum.

wurde die Substanz mit $\mathrm{KMnO}_{4}$ in Schwefelsäure oxydiert ${ }^{12}$. Nach Aufarbeitung des Reaktionsgemisches fiel ein Produkt an, das papierchromatographisch und ionophoretisch eindeutig als Asparaginsäure identifiziert werden konnte. Tyrosin liefert unter gleichen Bedingungen ebenfalls Asparaginsäure. Die Vermutung lag nahe, daß die Verbindung $m$-Hydroxyphenylalanin ( $m$ Tyrosin) ist.

Die Substanz ist optisch aktiv: $[\alpha]{ }_{D}^{22}=-14,5^{\circ}$ (70-proz. Äthanol), $M_{\mathrm{D}}=-26^{\circ} ;[\alpha] \frac{22}{\mathrm{D}}=+8,9^{\circ}$ (70-proz. Äthanol, 2-n. an $\mathrm{HCl}), M_{\mathrm{D}}=+16^{\circ}$. Nach der Regel von Lutz und Jirgenson ${ }^{\mathbf{1 3}}$ zeigen Aminosäuren der L-Reihe in saurem Milieu Abnahme der negativen bzw. Zunahme der positiven Rotation. Danach sollte die Verbindung der L-Reihe angehören und die vollständige Bezeichnung L- $(-)-m$-Tyrosin lauten.

Synthetisches $m$-Tyrosin * (Schmp. $267-270{ }^{\circ} \mathrm{C}$, Zersetzung) zeigt papierchromatographisch völlige Übereinstimmung mit der von uns isolierten Verbindung. Die UV-Absorptionsspektren sind ebenfalls identisch. Acetylierung auf dem Papier ${ }^{14}$ führte $\mathrm{zu}$ einem $N$-Acetylderivat, das für beide Verbindungen gleiches chromatographisches Verhalten zeigt. Bei Papierchromatographie erweist sich $m$-Tyrosin als recht variabel und reagiert mit Ninhydrin einmal zu einem rötlichvioletten, zum anderen zu einem gelben Farbstoff. Der Ausfall der Reaktion scheint stark vom Laufmittel abhängig zu sein; ähnlich verhält es sich mit der Fluoreszenz im UV-Licht, die in einigen Laufmitteln

* Herrn Dr. M. Luckner, Pharmakognostisches Institut der Universität Halle, danken wir für UUberlassung einer kleinen Probe $m$-Tyrosin.

12 G. Schneider, Biochem. Z. 330, 428 [1958].

13 O. Lutz u. B. Jirgenson, Ber. dtsch. chem. Ges. 63, 448 [1930]; 64, 1221 [1931]; 65, 784 [1932].

14 F. Kuffner u. T. Kirchenmayer, Mh. Chem. 92, 701 [1961]. gelöscht ist. Im Zusammenhang damit sind besonders in phenolhaltigen Laufmitteln gelegentlich starke $R_{f}$ Wert-Verschiebungen zu beobachten. Wir führen dieses unnormale Verhalten auf die $m$-ständige OH-Gruppe zurück, die oxydativen Angriffen leichter zugänglich ist als eine $p$-ständige. Möglicherweise ist auch mit der Ausbildung von Wasserstoffbrücken zwischen $\mathrm{OH}$ - und $\mathrm{NH}_{2}$-Gruppe zu rechnen.

Im Milchsaft von Euphorbia myrsinites L. findet sich neben $m$-Tyrosin in geringerer Menge eine zweite Verbindung (Schmp. 270-271 ${ }^{\circ} \mathrm{C}$, Zersetzung), die nicht mit Ninhydrin reagiert und für die wir auf Grund der bisher vorliegenden Befunde die Struktur eines $N$-substituierten $m$-Tyrosins annehmen. Dem $m$-Tyrosin nahestehende Verbindungen mit wenigstens gleicher Ringsubstitution fanden wir auch in den Milchsäften von Euphorbia helioscopia L. und Euphorbia tirucalli L.

Über das Vorkommen von $m$-Tyrosin in freier Form in der Natur ist unseres Wissens noch nichts berichtet worden. Die Verbindung scheint aber für die Biosynthese des Viridicatols ${ }^{15}$ aus Penicillium viridicatum und des Gliotoxins ${ }^{16}$ aus Aspergillus-Arten von Bedeutung zu sein.

In letzter Zeit wurden mehrere ungewöhnliche aromatische Aminosäuren aus Pflanzen isoliert, so das 2.4Dihydroxy-6-methyl-phenylalanin aus den Samen von Agrostemma githago ${ }^{12}$, das $m$-Carboxy-phenylglycin und das $m$-Carboxy-phenylalanin aus dem Rhizom von Iris tingitana ${ }^{17}$, zu denen jetzt $m$-Tyrosin hinzukommt.

15 J. H. Birkinshaw, M. Luckner, Y. S. Mohammed, K. Mothes u. C. E. Stickings, Biochem. J. 89, 196 [1963].

16 M. R. Bell, J. R. Johnson, B. S. Wildi u. R. B. Woodward, J. Amer. chem. Soc. 80, 1001 [1958].

17 C. J. Morris, J. F. Thompson, S. Asen u. F. Irreverre, J. Amer. chem. Soc. 81, 6069 [1959] ; J. biol. Chemistry 236. 1181 [1961]. 